\title{
Deciphering the complexity of macrophage biology and function with the zebrafish model
}

\author{
Nguyen-Chi Mai $^{1,2}$, Laplace-Builhé Béryl ${ }^{1,2}$, Jorgensen Christian ${ }^{1,2,3}$, Farida Djouad ${ }^{1,2}$ \\ ${ }^{1}$ Inserm U 1183, IRMB, Montpellier, 34295 France \\ ${ }^{2}$ Université de Montpellier, Montpellier, 34295 France \\ ${ }^{3}$ Clinical unit for osteoarticular diseases and Department for Biotherapy, CHU Lapeyronie, Montpellier, 34295, France
}

Correspondence: Farida Djouad

E-mail: farida.djouad@inserm.fr

Received: March 17, 2016

Published online: May 03, 2016

\begin{abstract}
Macrophages are present in every tissue of the body. Acting as sentinels of the immune system, they have the ability to engulf apoptotic cells and pathogens and also produce effector signals to mount a proper immune response. In response to tissue injury or microbe invasion, they are rapidly recruited to the injured site and are main key players in the protection of the host. Associated with many diseases, macrophages can have deleterious roles, especially due to their pro-inflammatory function. However, macrophages were discovered to be central during the orchestration of every step of inflammation, from initiation to resolution phases and to participate in tissue development, homeostasis and repair. These plural functions exerted by macrophages support their high plasticity and versatility potential and suggest the existence of macrophage subsets that might be differentially activated and/or recruited during physiological and pathological processes. The study of macrophage behaviour dynamics and functions in their activated states requires tractable in vivo models in which macrophages can be visualized under physiological or stress conditions. Thus, the zebrafish (Danio rerio) has emerged as an excellent vertebrate system to study myeloid development, inflammation and host-pathogen interactions. Conservation of immune cell lineage, optical accessibility of the zebrafish embryo combined with genetic approaches unable us to improve our understanding of macrophage functions. In this review, we discuss recent studies that have used the zebrafish advantages to provide key advances in the field of vertebrate macrophage biology and functions.
\end{abstract}

Keywords: Zebrafish; macrophage; inflammation; polarization; infection

To cite this article: Nguyen-Chi Mai, et al. Deciphering the complexity of macrophage biology and function with the zebrafish model. Macrophage 2016; 3: e1260. doi: 10.14800/ Macrophage.1260.

Copyright: (C) 2016 The Authors. Licensed under a Creative Commons Attribution 4.0 International License which allows users including authors of articles to copy and redistribute the material in any medium or format, in addition to remix, transform, and build upon the material for any purpose, even commercially, as long as the author and original source are properly cited or credited.

\section{Introduction}

Initially described for their phagocytic capacities, macrophages play a central role in inflammation and belong to the first line of defense in the case of wound or infection. The presence of macrophage during tissue repair has led to identify macrophage involvement in the orchestration of inflammation and its resolution and thus in the restoration of tissue homeostasis and wound healing ${ }^{[1,2]}$. Macrophages are also involved in many pathological processes, appearing therefore as critical therapeutic targets for controlling disease progression. Therefore, innovative strategies which seek to deplete macrophages or modulate their functions could be developed in order to alter the outcome of the diseases. 
Table 1. Tools and zebrafish lines to study macrophage in zebrafish model system

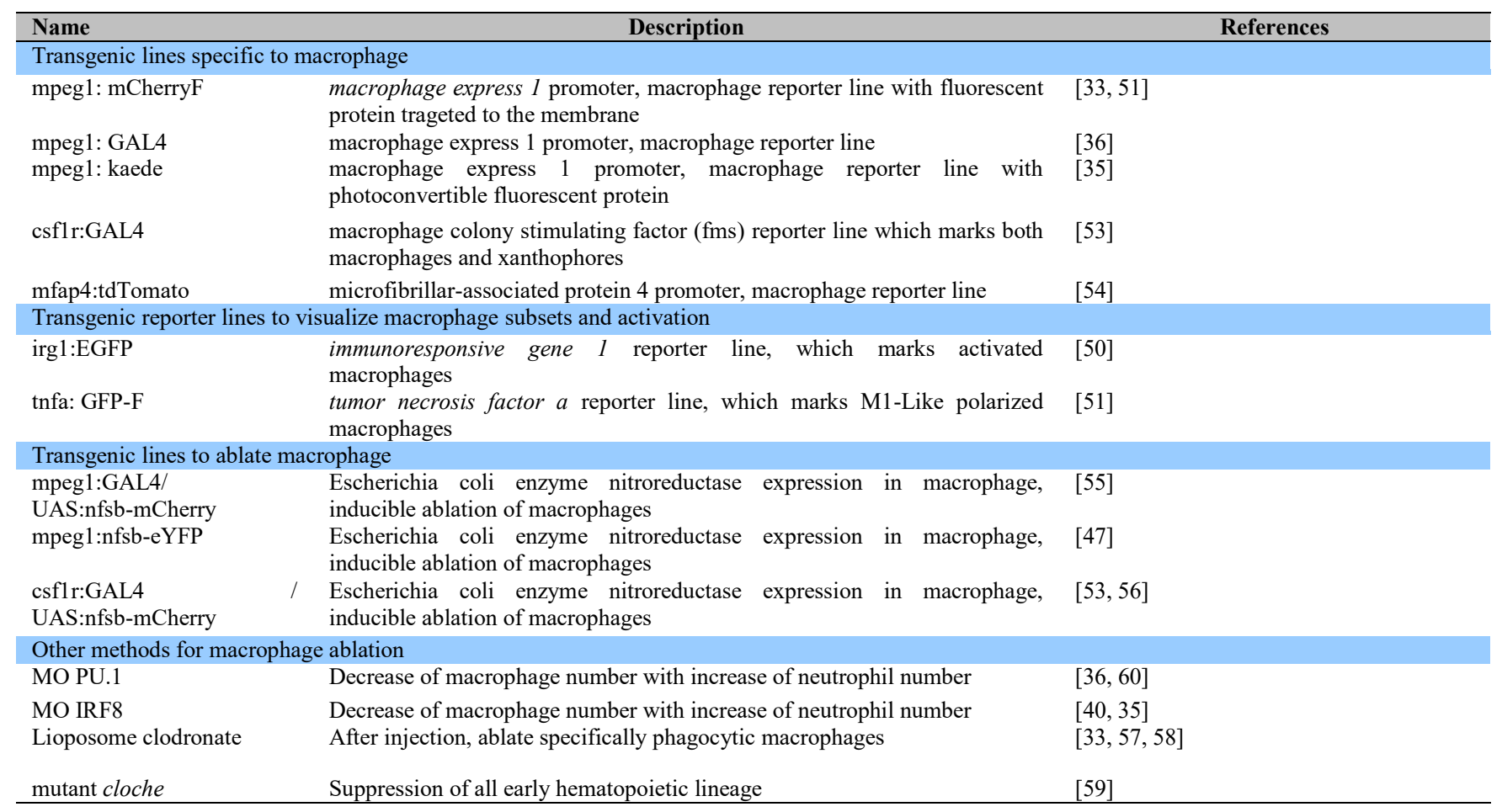

To investigate the complexity of the role of macrophages, several mammalian models have been developed. Indeed, the establishment of transgenic mouse strains allowed macrophage tracing and depletion thus improving our knowledge on their functions in tissue homeostasis and diseases. In contrast to zebrafish and other lower vertebrates, mouse and human macrophages have been extensively studied in vitro mostly using monocyte-derived macrophages and are thus well characterized ${ }^{[3-5]}$. According to the stimuli encountered by macrophages, they adopt different phenotypes and functions by a process called activation or polarization. They were thus classified into subpopulations according to their activation state. Classically activated macrophages (M1) produce toxic molecules such as inflammatory cytokines and reactive oxygen/nitrogen species and are involved in the resistance against intracellular parasites and tumor cells. The alternative macrophages (M2) participate in the resolution of inflammation, parasite clearance and tissue remodeling [4, 6, 7]. Macrophage polarization into the M1 phenotype required T helper type 1 (Th1) cytokines and LPS, which stimulate their capacity to secrete TNF, IL12 and IL-23. The exposition of macrophages to cytokines such as IL-4 induces their polarization toward a M2 phenotype producing large amount of IL-10 combined with high levels of scavenger, mannose and galactose-type receptors ${ }^{[4]}$. Although simplistic to apprehend the complexity of macrophages, this classification was established to better understand their plasticity and functions. Therefore, this designation does not really reflect macrophage diversity in vivo ${ }^{[3,8]}$. Indeed, observations of macrophages in chronic inflammation, cancer or infection reveal that this cell population display a much larger transcriptional repertoire according the set of stimuli they received enlarging the binary M1/M2 macrophage polarization concept to a spectrum model ${ }^{[3]}$. The study of macrophage functions requires in vivo tractable systems in which macrophage diversity could be captured during physiological or pathological conditions in an entire organism.

The zebrafish has emerged as an exquisite vertebrate model system for leukocyte biology studies. Indeed, this little tropical fish possesses many advantages including 1) ex vivo development making zebrafish embryos easy to visualize, 2) the transparency of its larvae allow to monitor immune cells in real time and in vivo, 3) the amenability to genetic manipulation and 4) the ease to cell lineage and pathogen tracing with specific color coding to monitor the dynamics of their behavior and interaction. These unique features of zebrafish provide an outstanding opportunity to get new insights into the field of macrophage biology and functions.

Zebrafish innate immunity shares many features with mammalian systems ${ }^{[9]}$. In particular, zebrafish macrophages harbor morphological, phenotypical, biochemical, and functional features similar to mammalian macrophages. The recent development of transgenic zebrafish displaying immune progenitors or macrophages specifically labeled with fluorescent proteins (Table 1) has led to key advances in 


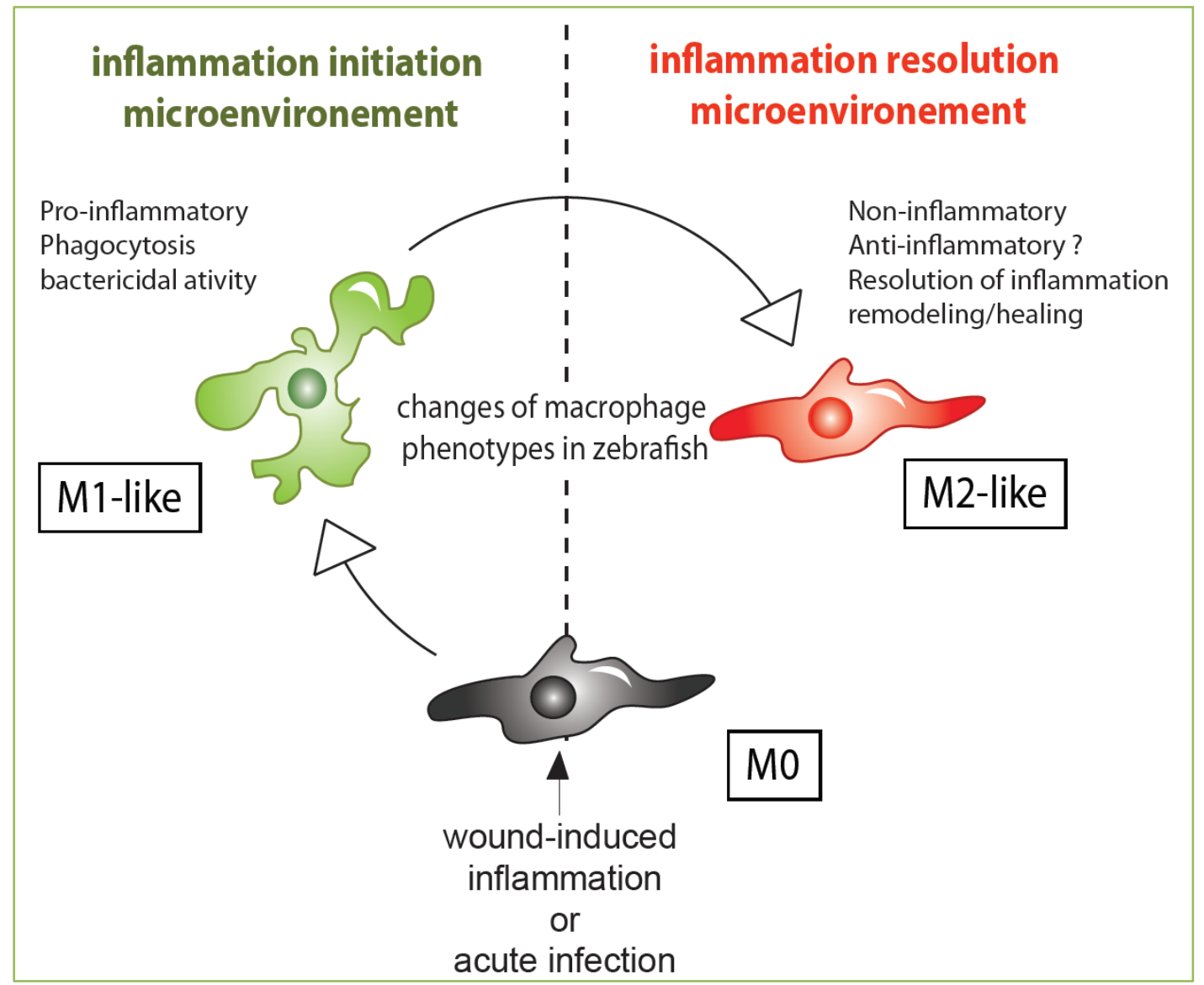

Figure 1. Hypothetical model for macrophage polarization and plasticity in response to wound induced inflammation or acute infection in zebrafish larvae. Diagram representing macrophage activation and polarization in zebrafish. Unpolarised macrophages (MO) are mobilised and recruited to the wound or the infection site. They are activated and polarized toward a M1-like phenotype (pro-inflammatory). In response to changes in environmental cues, the same macrophages progressively change their phenotype toward intermediate phenotypes and maybe fully polarized M2-like phenotype (non -inflammatory).

both septic and aseptic contexts such as in the field of wound healing and host/pathogen interactions, respectively. This review highlights some key recent studies that have used the zebrafish model to provide new insights into vertebrate macrophage biology and functions. More exhaustive review on hematopoiesis, immunity and host-pathogen interactions in zebrafish have already been published ${ }^{[10-15]}$.

\section{Macrophage ontogeny in zebrafish}

Macrophages are generated during hematopoiesis, a process that occurs in distinct waves during embryonic development and adulthood called primitive and definitive hematopoiesis. In human and mouse, primitive macrophages originate from erythro-myeloid progenitors in the blood islands of the yolk sac of the embryo. By contrast, zebrafish early hematopoiesis is recapitulated in two anatomically distinct sites. Specification of primitive erythrocytes is driven by gata1 in the posterior mesoderm that will become the intermediate cell mass. Between 12 and 24 hours post fertilization (hpf), primitive macrophage progenitors emerge rostrally from the lateral plate mesoderm (anterior to the cardiac field) in a spi-1/pu. 1 expression dependent manner ${ }^{[16,}$ 17]. Primitive macrophages then invade the embryo (the mesenchyme of the head, blood circulation, etc). The migration of primitive macrophages in the embryonic tissues and subsequent microglia formation in the head require the expression of M-CSF receptor/fms/csflr gene ${ }^{[18,19]}$. Then, from 26 to $48 \mathrm{hpf}$, a transient wave of definitive hematopoiesis takes place in blood islands with the specification of multipotent erythro-myeloid progenitors. These $1 \mathrm{mo}^{+}$gata $1^{+}$progenitor cells that display a limited proliferative potential will give rise to erythrocytes and myeloid cells ${ }^{[20]}$.

In mammals, definitive hematopoiesis initiates in the aorta-gonad-mesonephros (AGM) region of the embryo ${ }^{[21]}$. In zebrafish, multipotent hematopoietic stem cells (HSCs) 
http://www.smartscitech.com/index.php/Macrophage

emerge around 26-28 hpf from the endothelium of the ventral wall of the aorta and seed an homologous tissue with the AGM of amniotes, between the dorsal aorta and axial vein and express in their undifferentiated state cd41, c-myb and runx 1 markers ${ }^{[22-24]}$. HSCs then migrate through the blood to the caudal hematopoiesis tissue (CHT) where they expand and differentiate to give rise to all hematopoietic lineages.

From 4 days post fertilization (dpf) HSCs located in the CHT further migrate through the circulation to colonize the major definitive hematopoietic organs i.e. the thymus and the kidney. HSCs of the kidney ensure macrophage supply for subsequent larval stages and adult life ${ }^{[24,25]}$. While it is well established that monocytes differentiate into macrophages during inflammation in response to a threat in mammals, monocytes populations have not yet been fully characterized in the zebrafish.

\section{Role of macrophages in host-pathogen interactions in zebrafish}

The zebrafish embryo has been a favorite model for developmental biologists. However, recently, the number of zebrafish infection models for pathogens has exploded. We will not address the multiple ways by which the immune system interacts with pathogens in zebrafish, as there are excellent reviews covering this subject ${ }^{[10-12]}$. Instead, we will focus exclusively on recent studies that have highlighted the role of macrophages in the context of infections, wound healing and regeneration in zebrafish larvae.

In vitro systems cannot fully recapitulate the behavior of leukocytes in 3D architecture organs and their dynamic interaction with microorganisms. A comparative analysis of the behavior of macrophages and neutrophils during early stages of E. coli infection was performed using high-resolution live imaging in zebrafish larvae ${ }^{[26]}$. This study revealed that microbe elimination by neutrophils and macrophages depends not only on the nature of the invading microbe, but also on the nature of the site of infection such as the surface, matrix, fluid etc... Indeed neutrophils efficiently engulf bacteria on tissue surfaces but are poorly efficient to phagocytose microbes in fluid environments. By contrast, macrophages are able to engulf microbes regardless of the infected organ or tissue ${ }^{[26]}$.

Tuberculosis (TB) is a chronic disease due to mycobacteria infection. It is one of the leading causes of death worldwide. While about one-third of world population is supposed to be infected with Mycobacterium tuberculosis (M. tuberculosis), 5-10\% of infected individuals develop active tuberculosis. In the other infected individuals, mycobacteria persist within granulomas as a dormant form.
Zebrafish models for tuberculosis using infection with Mycobacterium marinum (M. marinum) have brought tremendous insights in the field and helped to understand the early phase of the disease. M. marinum is a natural pathogen of the fish and M. marinum and M. tuberculosis are closely related genetically. When infecting the fish, M. marinum leads to a physiopathology very closed to the human TB, including the formation of granulomas. Due to its many advantages, the zebrafish larva offers an outstanding opportunity to unravel early stages of granuloma formation and the role of macrophages in this process. Adaptive immune system of the zebrafish requires several weeks of development to become functional. Therefore embryos and young larvae only possess an innate immune system ${ }^{[27]}$.

Davis and collaborators ${ }^{[28]}$ showed that despite the absence of adaptive immune system, M. marinum infection of zebrafish embryos leads to granulomas formation within few days, showing that macrophage-mycobacteria interaction is sufficient to initiate granuloma formation in the context of innate immunity. The recruitment of new macrophages to nascent granulomas and their motility throughout the structure are dependent on the ESX-1/RD1 virulence locus ${ }^{[29]}$. Using a M. marinum strain expressing Kaede photoactivatable protein, infected macrophages were tracked demonstrating that newly infected macrophages from primary granulomas seed secondary granulomas. While granulomas were believed to benefit the host by containing and restricting mycobacteria, this study shows that granulomas participate to the expansion and dissemination of early tuberculous infection via infected macrophages ${ }^{[29]}$.

TB relies on the ability of mycobacteria to invade the host from peripheral infection site in the distal lung airways to deeper tissues. Because infected macrophages play a major role in the transport of the mycobacteria, many research focused on how $M$. tuberculosis survive or escape microbicidal activity of these phagocytes. Infection in the hindbrain ventricle of zebrafish larvae with $M$. marinum was shown to lead to the recruitment and the infection of CCR2 ${ }^{+}$ iNos ${ }^{-}$macrophages which possess a low microbicidal activity and are thus called "permissive" ${ }^{[30]}$. One of the mechanisms by which macrophages sense pathogenic microorganisms in mammals and in zebrafish is through the recognition of pathogen molecular patterns (PAMPs) that are present in microbial components with Toll-like receptors (TLRs) ${ }^{[9,31}$ ${ }^{32}$. However, in this context of infection, pathogenic M. marinum sensing is largely independent of myd88, a central regulator of TLRs. This immune evasion is linked to the presence of phthiocerol dimycocerosate (PDIM) lipids on the bacteria cell-surface that mask PAMPs. By contrast, infection with $M$. marinum mutant that lacks PDIM (DmmpL7) leads to the recruitment of microbicidal 
macrophages (CCR2- iNos $^{+}$) in a TLR/Myd88 dependent pathway. Interestingly, co-infection with M. marinum together with $\mathrm{P}$. aeruginosa or $\mathrm{S}$. aureus, two bacterial colonizers of the pharynx that induce TLR signaling lead to the attenuation of M. marinum. This elegant study highlights a new mechanism by which PDIM-mediated evasion of microbicidal macrophages and suggests a central role for commensal flora in controlling mycobacterial entry ${ }^{[30]}$.

The importance of macrophage supply and demand in granuloma fate was recently highlighted using M. marinum infected embryo system ${ }^{[19]}$. Indeed, during the first phase of granuloma growth, a macrophage demand leads to the recruitment of new macrophages. But when macrophage global pool becomes limited, macrophage supply stops leading to granuloma necrosis and increase of bacteria burden. Csfr1 plays a major role in the replenishment of macrophage pool and thus in macrophage supply. Inversely, increasing macrophage supply to the granuloma delays the process of granuloma necrosis. Therefore, this study reveals that macrophage supply boost with myeloid growth factors might be a host-targeting therapy for $\mathrm{TB}^{[19]}$.

Interestingly, a recent work on Mycobacterium abscessus (M. abscessus) infection in zebrafish larvae have shown a role of macrophages in the transport of the bacteria to the central nervous system (CNS) ${ }^{[33]}$. M. abscessus is responsible for severe pulmonary infections in cystic fibrosis patients. Systemic infection with the virulent rough variant in the zebrafish larvae leads to the formation of particular bacterial structures called cords, in the vasculature and in the CNS where they initiate abscess. Importantly, macrophage depletion experiments showed that macrophages play a major role in the dissemination of $\mathrm{M}$. abscessus into the host tissues, especially in the CNS and the restriction of mycobacterial cording. Finally, cords prevent phagocytosis by neutrophils and macrophages most probably due to their large size highlighting thus a new mechanism of immune evasion ${ }^{[33]}$.

\section{Role of macrophages in wound healing}

Wound healing is an essential biological process relying on conserved mechanisms whose aim is to restore barrier integrity and tissue homeostasis. After wounding, chemoattractant molecules which include the contents of damaged cells and chemokines produced by intact cells around the wound are released. The reactive oxygen species $\mathrm{H}_{2} \mathrm{O}_{2}$ were shown to play an immediate role as a chemoattractant in zebrafish ${ }^{[34]}$. Indeed using zebrafish larvae expressing a genetically encoded $\mathrm{H}_{2} \mathrm{O}_{2}$ sensor called HyPer, which detects intracellular $\mathrm{H}_{2} \mathrm{O}_{2}$, the authors showed that $\mathrm{H}_{2} \mathrm{O}_{2}$ forms a chemotactic gradient in the first minutes following caudal fin injury. This gradient of $\mathrm{H}_{2} \mathrm{O}_{2}$, produced from the edge of the wound through the NADPH oxydase DUOX activity, was shown to recruit neutrophils, the other well represented phagocyte population ${ }^{[34]}$. In Drosophila melanogaster, a similar gradient which recruits the macrophage-like cells called haemocytes has been observed after laser wounding, suggesting that $\mathrm{H}_{2} \mathrm{O}_{2}$ gradient might be an evolutionary conserved danger signal. In zebrafish, the $\mathrm{H}_{2} \mathrm{O}_{2}$ gradient not only recruits neutrophils at the wound but also macrophages. Macrophage migration toward the wound site is dependent of the SRC family Kinase, Yes-related kinase that may acts as a sensor for $\mathrm{H}_{2} \mathrm{O}_{2}{ }^{[35]}$.

The in vivo study of respective behaviour of the different phagocyte populations in response to wounding has been considerably improved by the use of genetic reporters which specifically marks with distinguishable fluorophores macrophages and neutrophils ${ }^{[36]}$. Indeed, neutrophils were shown to migrate faster than macrophages, the latter taking a more direct route to the wound margin. After arrival at the wound, neutrophils display a roaming behaviour while macrophages stay at the wound site ${ }^{[36]}$. By contrast to macrophages which remain at the wound up to 4 days, neutrophils undergo reverse migration from 6 hours post injury ${ }^{[37]}$. Furthermore, interactions between neutrophil and macrophage in vivo were analysed during wounding revealing phagocytosis of apoptotic neutrophils by macrophages to initiate inflammation resolution phase. This process was dynamically observed in zebrafish. Direct contacts between macrophages and living neutrophils resulting in some cases with the transfer of cytoplasm portions from neutrophil to macrophage were also shown ${ }^{[36]}$. More recently, macrophages-neutrophils direct interactions in response to tissue injury were described to promote neutrophilic resolution through a process of neutrophil reverse migration ${ }^{[35]}$.

\section{Role of macrophages during zebrafish caudal fin regeneration}

Regeneration is a sophisticated and coordinated process which consists in the complete restoration of the structure, the mass and the function of the injured tissue/organ. In most adult vertebrates the regeneration capacity is limited to few organs, such as liver, skin, muscle and bones. However, it is possible to observe appendage regeneration in some amphibian and fish species. This phenomenon requires the complete cooperation and tight coordination of numerous cell populations including immune cells such as macrophages which rapidly invade the wound to orchestrate the inflammatory response. Somatic cells from injured tissues as well as stem cells also contribute to the establishment of the new tissue through the formation of a 
highly proliferative structure called the blastema ${ }^{[38-41]}$. Several evidences have underlined the central and conserved role of inflammation and macrophages in this process. After an injury, although the inflammatory response is required for limiting the area of tissue damage and debris clearance, it might be deleterious if it persists during the whole process of tissue repair. Indeed, in larval Xenopus which is known to regenerate only during its early developmental stages, hindlimb amputation triggers a transient inflammatory response followed by an increased expression of blastemal markers that signs the regeneration process ${ }^{[42]}$. In contrast, when the limb amputation is performed after metamorphosis it results in a persistent inflammatory response marked by the absence of blastemal marker expression. Moreover, while chronic inflammation during the regeneration competent stages leads to a dramatic decrease of the regenerative capacity of the Xenopus, the inhibition of the continual inflammation in the incompetent stages improves its regenerative ability. Therefore, the transient and tightly controlled inflammatory response is needed for a successful regeneration as its disruption leads to imperfect regeneration. These results in anurans also underline a nuanced role of the cellular actors of inflammation during regeneration.

The critical role of macrophages in the regeneration process has also been demonstrated in the well-known regeneration model; the salamander. Indeed, systemic macrophage depletion inhibits the limb regeneration ability of the axolotl associated with massive fibrosis ${ }^{[43]}$. These findings strongly exhibit the positive role of macrophages in adult amphibian regeneration process underlying the importance of their activation, function and regulation for the outcome of wound resolution and regeneration.

However, in zebrafish larvae, morpholino strategy to study the role of macrophages in the context of caudal fin regeneration led to controversial results. Indeed, while the fin regeneration process was not altered by blocking the development of all myeloid lineage using Pu.1 morpholino (MO), the inhibition of the differentiation of macrophage lineage using interferon regulatory factor 8 (irf8) MO delayed or did not affect this process ${ }^{[40,44,45]}$. In zebrafish cloche mutants, characterized by the lack of most hematopoietic tissues, the regeneration is initiated after caudal fin amputation but failed because of the loss of regenerative cell proliferative potential ${ }^{[46]}$. These controversies might be explained by the lack of specific tools to deplete the macrophage lineage without altering the generation of other cell types and also point out the need for developing strategies to accurately define the role of macrophages during regeneration. In adult zebrafish, while macrophage depletion during the whole regeneration process alters the potential of fin regeneration by affecting the proliferative capacity of blastema cell, their depletion during the tissue outgrowth phase only modify the pattern of the newly formed tissue ${ }^{[47]}$. These differential roles exerted by macrophages during epimorphic regeneration suggest the sequential recruitment of macrophage subtypes that govern the different phases of appendage regeneration.

\section{Macrophage polarization in zebrafish}

Macrophages subpopulations have been intensively studied in vitro and result from activation processes. While results obtained from in vitro studies suggest the possible phenotype skewing of macrophages from M1 to M2, a recent study argues for the sequential homing of M1 and M2 macrophages to the site of inflammation $[1,48,49]$. This controversy underlined the need for accurate real-time tracing of macrophage sub-populations in vivo in response to various environmental cues. The existence of macrophage subtypes in zebrafish has been recently addressed using transgenic reporter zebrafish lines reporting the activated states of macrophages in response to stress [50, 51]. Immunoresponsive gene 1 (irg1) encodes a mitochondria-localizing enzyme that is involved in bactericidal activity of Macrophages. Irg1 is specifically induced in macrophages following injection of live Salmonella enterica serovar Typhimurium into the hindbrain ventricle, showing that irg1 is a responsive gene to macrophage activation ${ }^{[52]}$. The transgenic line Tg(irg1:EGFP) in which EGFP expression is driven by irg1 promoter, can be combined with transgenic lines that constitutively mark macrophages, allowing thus real time visualization of macrophage activation in response to LPS and xenografted human cancer cells (Table 1) ${ }^{[50]}$. Going further, we recently generated new transgenic reporter zebrafish line in which macrophages positive for tnfa, a key feature of classically activated M1 express both eGFP and mCherry farnelysated fluorescent proteins: $\operatorname{Tg}(\mathrm{mpeg} 1: \mathrm{mCherry}-\mathrm{F} / \mathrm{tnfa}$ GFP-F). This line allowed us to track activated macrophages during the multi-step inflammation process induce by wounding and during infection. Injection in the muscle of live E. coli expressing E2-crimson fluorescent protein leads to the recruitment of macrophages and their subsequent activation while engulfing fluorescent bacteria ${ }^{[51]}$. We showed that caudal fin amputation-mediated inflammation triggered macrophage recruitment, some of which started to adopt a M1-like phenotype expressing tnfa. Cell sorted tnfa ${ }^{+}$and tnfa polarized macrophages activated during the regeneration process, respectively expressed some mammalian markers of M1 and M2 macrophages. During the time-course of inflammation, pro-inflammatory macrophages converted into M2-like phenotype ${ }^{[51]}$. Live imaging of zebrafish larvae permitted the first real time visualization of macrophage activation and polarization demonstrating the diversity and 
http://www.smartscitech.com/index.php/Macrophage

plasticity of zebrafish macrophage subsets. The molecular analysis of these macrophage subtypes highlighted the evolutionary conservation of macrophage subpopulations from fish to mammals ${ }^{[51]}$. Further development of novel inducible reporter lines in combination with established macrophage fluorescent transgenic lines (Table 1), will help to understand the relationship between activation programs and macrophage functions.

\section{Conclusions}

These important findings demonstrate that work in tractable vertebrate systems, as the zebrafish, can be highly informative for the study of myeloid biology owing to the conservation of innate immune lineage between zebrafish and human. Live imaging of transparent transgenic zebrafish larvae had allowed the visualization of macrophage migration, interactions and activation/polarization after tissue injury and infections demonstrating the strength of this model to track macrophage functions and fate in pathological situations. For these reasons, we can expect more use of zebrafish in the future. The establishment of new zebrafish transgenic lines should improve our understanding of the molecular pathway associated with macrophage functions. In addition, in the field of pharmacological research, small molecule screening using the zebrafish embryo may lead to the identification of new therapeutic compounds.

\section{Conflicting interests}

The authors have declared that no conflict of interests exist.

\section{References}

1. Sica A, Mantovani A. Macrophage plasticity and polarization: in vivo veritas. J Clin Invest 2012; 122:787-795.

2. Pollard JW. Trophic macrophages in development and disease. Nat Rev Immunol 2009; 9:259-270.

3. Xue J, Schmidt SV, Sander J, Draffehn A, Krebs W, Quester I, et al. Transcriptome-based network analysis reveals a spectrum model of human macrophage activation. Immunity 2014; 40:274-288.

4. Italiani P, Boraschi D. New Insights Into Tissue Macrophages: From Their Origin to the Development of Memory. Immune Netw 2015; 15:167-176.

5. Wynn TA, Chawla A, Pollard JW. Macrophage biology in development, homeostasis and disease. Nature 2013; 496:445-455.

6. Chazaud B. Macrophages: supportive cells for tissue repair and regeneration. Immunobiology 2014; 219:172-178.

7. Gordon S, Pluddemann A, Martinez Estrada F. Macrophage heterogeneity in tissues: phenotypic diversity and functions. Immunol Rev 2014; 262:36-55.

8. Martinez FO, Helming L, Milde R, Varin A, Melgert BN, Draijer $\mathrm{C}$, et al. Genetic programs expressed in resting and IL-4 alternatively activated mouse and human macrophages: similarities and differences. Blood 2013; 121:e57-69.

9. van der Vaart M, Spaink HP, Meijer AH. Pathogen recognition and activation of the innate immune response in zebrafish. Adv Hematol 2012; 2012:159807.

10. Torraca V, Masud S, Spaink HP, Meijer AH. Macrophage-pathogen interactions in infectious diseases: new therapeutic insights from the zebrafish host model. Dis Model Mech 2014; 7:785-797.

11. Levraud JP, Palha N, Langevin C, Boudinot P. Through the looking glass: witnessing host-virus interplay in zebrafish. Trends Microbiol 2014; 22:490-497.

12. Meijer AH, Spaink HP. Host-pathogen interactions made transparent with the zebrafish model. Curr Drug Targets 2011; 12:1000-1017.

13. Ellett F, Lieschke GJ. Zebrafish as a model for vertebrate hematopoiesis. Curr Opin Pharmacol 2010; 10:563-570.

14. Renshaw SA, Trede NS. A model 450 million years in the making: zebrafish and vertebrate immunity. Dis Model Mech 2012; 5:38-47.

15. Forrester AM, Berman JN, Payne EM. Myelopoiesis and myeloid leukaemogenesis in the zebrafish. Adv Hematol 2012; 2012:358518.

16. Herbomel P, Thisse B, Thisse C. Ontogeny and behaviour of early macrophages in the zebrafish embryo. Development 1999; 126:3735-3745.

17. Lieschke GJ, Oates AC, Paw BH, Thompson MA, Hall NE, Ward $\mathrm{AC}$, et al. Zebrafish SPI-1 (PU.1) marks a site of myeloid development independent of primitive erythropoiesis: implications for axial patterning. Dev Biol 2002; 246:274-295.

18. Herbomel P, Thisse B, Thisse C. Zebrafish early macrophages colonize cephalic mesenchyme and developing brain, retina, and epidermis through a M-CSF receptor-dependent invasive process. Dev Biol 2001; 238:274-288.

19. Pagan AJ, Yang CT, Cameron J, Swaim LE, Ellett F, Lieschke GJ, et al. Myeloid Growth Factors Promote Resistance to Mycobacterial Infection by Curtailing Granuloma Necrosis through Macrophage Replenishment. Cell Host Microbe 2015; $18: 15-26$.

20. Bertrand JY, Kim AD, Violette EP, Stachura DL, Cisson JL, Traver D. Definitive hematopoiesis initiates through a committed erythromyeloid progenitor in the zebrafish embryo. Development 2007; 134:4147-4156.

21. Dzierzak E, Speck NA. Of lineage and legacy: the development of mammalian hematopoietic stem cells. Nat Immunol 2008; 9:129-136.

22. Kissa K, Herbomel P. Blood stem cells emerge from aortic endothelium by a novel type of cell transition. Nature 2010; 464:112-115.

23. Bertrand JY, Chi NC, Santoso B, Teng S, Stainier DY, Traver D. Haematopoietic stem cells derive directly from aortic endothelium during development. Nature 2010; 464:108-111.

24. Murayama E, Kissa K, Zapata A, Mordelet E, Briolat V, Lin HF, et al. Tracing hematopoietic precursor migration to successive hematopoietic organs during zebrafish development. Immunity 2006; 25:963-975.

25. Kissa K, Murayama E, Zapata A, Cortes A, Perret E, Machu C, et $a l$. Live imaging of emerging hematopoietic stem cells and early thymus colonization. Blood 2008; 111:1147-1156.

26. Colucci-Guyon E, Tinevez JY, Renshaw SA, Herbomel P. Strategies of professional phagocytes in vivo: unlike macrophages, neutrophils engulf only surface-associated microbes. J Cell Sci 
http://www.smartscitech.com/index.php/Macrophage

\section{$2011 ; 124: 3053-3059$.}

27. Lam SH, Chua HL, Gong Z, Lam TJ, Sin YM. Development and maturation of the immune system in zebrafish, Danio rerio: a gene expression profiling, in situ hybridization and immunological study. Dev Comp Immunol 2004; 28:9-28.

28. Davis JM, Clay H, Lewis JL, Ghori N, Herbomel P, Ramakrishnan L. Real-time visualization of mycobacterium-macrophage interactions leading to initiation of granuloma formation in zebrafish embryos. Immunity 2002; 17:693-702.

29. Davis JM, Ramakrishnan L. The role of the granuloma in expansion and dissemination of early tuberculous infection. Cell 2009; 136:37-49.

30. Cambier CJ, Takaki KK, Larson RP, Hernandez RE, Tobin DM, Urdahl KB, et al. Mycobacteria manipulate macrophage recruitment through coordinated use of membrane lipids. Nature 2014; 505:218-222.

31. Akira S, Takeda K. Toll-like receptor signalling. Nat Rev Immunol 2004; 4:499-511.

32. van der Vaart M, van Soest JJ, Spaink HP, Meijer AH. Functional analysis of a zebrafish myd88 mutant identifies key transcriptional components of the innate immune system. Dis Model Mech 2013; 6:841-854.

33. Bernut A, Herrmann JL, Kissa K, Dubremetz JF, Gaillard JL, Lutfalla $\mathrm{G}$, et al. Mycobacterium abscessus cording prevents phagocytosis and promotes abscess formation. Proc Natl Acad Sci U S A 2014; 111:E943-952.

34. Niethammer P, Grabher C, Look AT, Mitchison TJ. A tissue-scale gradient of hydrogen peroxide mediates rapid wound detection in zebrafish. Nature 2009; 459:996-999.

35. Tauzin S, Starnes TW, Becker FB, Lam PY, Huttenlocher A. Redox and Src family kinase signaling control leukocyte wound attraction and neutrophil reverse migration. J Cell Biol 2014;207:589-598.

36. Ellett F, Pase L, Hayman JW, Andrianopoulos A, Lieschke GJ. mpeg1 promoter transgenes direct macrophage-lineage expression in zebrafish. Blood 2011; 117:e49-56.

37. Mathias JR, Perrin BJ, Liu TX, Kanki J, Look AT, Huttenlocher A. Resolution of inflammation by retrograde chemotaxis of neutrophils in transgenic zebrafish. J Leukoc Biol 2006; 80:1281-1288

38. Martin P, Leibovich SJ. Inflammatory cells during wound repair: the good, the bad and the ugly. Trends Cell Biol 2005; 15:599-607.

39. Arnold L, Henry A, Poron F, Baba-Amer Y, van Rooijen N, Plonquet A, et al. Inflammatory monocytes recruited after skeletal muscle injury switch into antiinflammatory macrophages to support myogenesis. J Exp Med 2007; 204:1057-1069.

40. Li L, Yan B, Shi YQ, Zhang WQ, Wen ZL. Live imaging reveals differing roles of macrophages and neutrophils during zebrafish tail fin regeneration. J Biol Chem 2012; 287:25353-25360.

41. London A, Itskovich E, Benhar I, Kalchenko V, Mack M, Jung S, et al. Neuroprotection and progenitor cell renewal in the injured adult murine retina requires healing monocyte-derived macrophages. J Exp Med 2011; 208:23-39.

42. Mescher AL, Neff AW, King MW. Changes in the inflammatory response to injury and its resolution during the loss of regenerative capacity in developing Xenopus limbs. PLoS One 2013; 8:e80477.

43. Godwin JW, Pinto AR, Rosenthal NA. Macrophages are required for adult salamander limb regeneration. Proc Natl Acad Sci U S A 2013; 110:9415-9420.

44. Mathew LK, Sengupta S, Kawakami A, Andreasen EA, Lohr CV,
Loynes CA, et al. Unraveling tissue regeneration pathways using chemical genetics. J Biol Chem 2007; 282:35202-35210.

45. LeBert DC, Squirrell JM, Rindy J, Broadbridge E, Lui Y, Zakrzewska A, et al. Matrix metalloproteinase 9 modulates collagen matrices and wound repair. Development 2015; 142:2136-2146.

46. Hasegawa T, Nakajima T, Ishida T, Kudo A, Kawakami A. A diffusible signal derived from hematopoietic cells supports the survival and proliferation of regenerative cells during zebrafish fin fold regeneration. Dev Biol 2015; 399:80-90.

47. Petrie TA, Strand NS, Yang CT, Rabinowitz JS, Moon RT. Macrophages modulate adult zebrafish tail fin regeneration. Development 2014; 141:2581-2591.

48. Porcheray F, Viaud S, Rimaniol AC, Leone C, Samah B, Dereuddre-Bosquet N, et al. Macrophage activation switching: an asset for the resolution of inflammation. Clin Exp Immunol 2005; 142(3):481-489.

49. Shechter R, Miller O, Yovel G, Rosenzweig N, London A, Ruckh $\mathrm{J}$, et al. Recruitment of beneficial M2 macrophages to injured spinal cord is orchestrated by remote brain choroid plexus. Immunity $2013 ; 38: 555-569$.

50. Sanderson LE, Chien AT, Astin JW, Crosier KE, Crosier PS, Hall CJ. An inducible transgene reports activation of macrophages in live zebrafish larvae. Dev Comp Immunol 2015; 53:63-69.

51. Nguyen-Chi M, Laplace-Builhe B, Travnickova J, Luz-Crawford $\mathrm{P}$, Tejedor $\mathrm{G}$, Phan QT, et al. Identification of polarized macrophage subsets in zebrafish. eLife 2015; 4:e07288.

52. Hall CJ, Boyle RH, Astin JW, Flores MV, Oehlers SH, Sanderson LE, et al. Immunoresponsive gene 1 augments bactericidal activity of macrophage-lineage cells by regulating beta-oxidation-dependent mitochondrial ROS production. Cell Metab 2013; 18:265-278.

53. Gray C, Loynes CA, Whyte MK, Crossman DC, Renshaw SA, Chico TJ. Simultaneous intravital imaging of macrophage and neutrophil behaviour during inflammation using a novel transgenic zebrafish. Thromb haemost 2011;105:811-819.

54. Walton EM, Cronan MR, Beerman RW, Tobin DM. The Macrophage-Specific Promoter mfap4 Allows Live, Long-Term Analysis of Macrophage Behavior during Mycobacterial Infection in Zebrafish. PloS one 2015;10:e0138949.

55. Palha N, Guivel-Benhassine F, Briolat V, Lutfalla G, Sourisseau $\mathrm{M}$, Ellett $\mathrm{F}$, et al. Real-time whole-body visualization of Chikungunya Virus infection and host interferon response in zebrafish. PLoS pathog 2013;9:e1003619.

56. Prajsnar TK, Hamilton R, Garcia-Lara J, McVicker G, Williams A, Boots $\mathrm{M}$, et al. A privileged intraphagocyte niche is responsible for disseminated infection of Staphylococcus aureus in a zebrafish model. Cell microbiol 2012;14:1600-1619.

57. Travnickova J, Tran Chau V, Julien E, Mateos-Langerak J, Gonzalez C, Lelievre E, et al. Primitive macrophages control HSPC mobilization and definitive haematopoiesis. Nat commun 2015;6:6227.

58. Van Rooijen N, Sanders A. Liposome mediated depletion of macrophages: mechanism of action, preparation of liposomes and applications. J immunol methods 1994;174:83-93.

59. Thompson MA, Ransom DG, Pratt SJ, MacLennan H, Kieran MW, Detrich HW, 3rd, et al. The cloche and spadetail genes differentially affect hematopoiesis and vasculogenesis. Dev biol 1998;197:248-269.

60. Rhodes J, Hagen A, Hsu K, Deng M, Liu TX, Look AT, et al. Interplay of pu. 1 and gata1 determines myelo-erythroid progenitor cell fate in zebrafish. Dev cell 2005;8:97-108. 